\title{
THE ROLE OF TEXTURE INFORMATION AND DATA FUSION IN TOPOGRAPHIC OBJECTS EXTRACTION FROM SATELLITE DATA
}

\author{
Kuldeep ${ }^{1}$, Pradeep Kumar GARG ${ }^{2}$ \\ Geomatics Engineering Group, Civil Engineering, \\ Indian institute of Technology Roorkee, India \\ E-mails: ${ }^{1}$ kchaurasia.nitb@gmail.com (corresponding author); ${ }^{2}$ pkgiitr@gmail.com
}

Received 4 June 2014; accepted 9 September 2014

\begin{abstract}
The growing availability of the satellite data has augmented the need of information extraction that can be utilized in various application including topographic map updation, city planning, pattern recognition and machine vision etc. The accurate information extraction from satellite images involves the integration of additional measures such as texture, shape etc. In this paper, investigation on extraction of topographic objects from satellite images by incorporating the texture information and data fusion has been made. The applicability of various texture measures based on the gray level co-occurrence matrix along with the effect of varying pixel window is also discussed. The classification results indicate that homogeneity texture image generated using $3 * 3$ window size is best suitable for topographic objects extraction. The best classification results with overall accuracy $85.0 \%$ and kappa coefficient 0.80 are obtained when classification is performed on fused image (Multispectral + PAN + Texture).
\end{abstract}

Keyworlds: texture descriptors, image classification, feature extraction, image fusion, topographic objects.

\section{Introduction}

Traditional methods employed in the classification of multispectral images generally use image spectral signature. It is commonly accepted in segmentation of spectrally homogeneous object classes because it is possible to outline fairly clean and representative training areas. However, outcome of such techniques are not satisfactory, especially in the applications involving the mapping of the manmade features and other inhomogeneous features in typical urban scenes. The outcomes of these methods are characterized by limited accuracy and low reliability (Haala, Brenner 1999). The reason behind this is that the urban objects are better recognized through their spatial properties rather than the spectral reflectance properties (Zhang 1999). In addition, it is very difficult to distinguish between two objects having high spectral similarity, such as roof of a building and pavements that are made using same material. Similarly, areas covered with green grass cannot be distinguished from tree.

The above mentioned traditional approach has poor performance in case of remote sensing data that has confined spectral potential such as SPOT and Cartosat-1 panchromatic images. This necessitates the enhancement of the data to support the classification of the different objects present in satellite image. In general, this can be done either by performing the analysis of data to expand the object feature space or by fusing it with some other data.

Data fusion approaches that incorporate texture information are very useful in various applications including image segmentation, image classification, land cover mapping and medical image analysis (Haack, Bechdol 2000; Rogova, Stomper 2002). Wu et al. (2007) suggested an approach for analyzing the color texture using multiple features data fusion method. They compared multiple features fusion and classification performance on the basis of the pyramid wavelet decomposition (PWD), incomplete tree-structured wavelet decomposition (ICTSWD) and wavelet packet decomposition (WPD). They found that multiple features fusion based on ICTSWD outperformed in classification performance and anti-noise ability as compared to PWD and WPD based fusion methods. Schistad and Jain (1997) applied various texture feature extraction 
methods to obtain multiple texture features and used these features to classify various objects in Synthetic Aperture Radar (SAR) imagery. Improved classification results were obtained.

High resolution satellite images are often used in applications where high quality segmentation results are expected. To obtain the high quality segmentation results, object feature base is required to be expanded that include textural, spectral and contextual features (Kiema 2002).

\section{Theoretical background}

Texture refers the frequency of tonal variation in an image. The first and second order statistics (co-occurrence matrices) are by far the most used statistical methods for texture discrimination. First order texture measures are one of the simplest descriptor that is more popular in image processing. Single pixel is used in calculation of gray level histogram. It indicates that the histogram consists of first order statistical information about the image. The shape of histogram plays a very important role in identifying the image characteristics. For example, a low-contrast image has the narrowly distributed shape of the histogram. If the image contains the object with a very narrow intensity range on a background of deffering intensity, will have the bimodal shape of the histogram (Srinivasan, Shobha 2008). Gray Level Co-occurrence Matrix (GLCM) is also known as Gray Tone Spatial Dependency (GTSD) matrix. The first order statistics uses original image values such as standard deviation but fails to consider the spatial relationship of neighbouring pixels. The second order statistics takes care of spatial relationship between groups of two pixels in original image. Since remote sensing images contain the abundant texture information of different type, which necessitates the texture extraction method that can capture spatial variation among pair of pixels.

Consider, an image $X$ of $M \times M$ dimension and $L$ gray levels is defined as:

$$
\{X(i, j), 0<I<M-1,0<j<M-1\} .
$$

In order to calculate the GLCM one must first define the window size, number of grey levels and offset direction. Where $\mathrm{L}$ is the number of grey levels considered, the GLCM will be an $L \times L$ matrix (Beliakov et al. 2008). GLCM texture considers the relation between two pixels at a time, called the reference and the neighbour pixel. The $L \times L$ Gray level co-occurrence matrix $W_{d}$ for a displacement vector $v=\left(v_{x}, v_{y}\right)$ is defined as:

$W_{d}(i, j)=\{((k, l),(m, n)): X(k, l)=i, X(m, n)=j\}$,

where each entry $(i, j)$ in matrix $W_{d}$ represents the number of occurrences of pair of gray levels $i$ and $j$ at distance $d$ apart. Consider an example of image $X$ of size $4 \times 4$ having 4 gray levels shown in Figure 1. GLCM for image I will be of size $4 \times 4$.

\begin{tabular}{|l|l|l|l|}
\hline 0 & 1 & 1 & 3 \\
\hline 0 & 0 & 1 & 1 \\
\hline 2 & 2 & 3 & 1 \\
\hline 2 & 2 & 3 & 3 \\
\hline
\end{tabular}

(a) Original image

\begin{tabular}{|l|l|l|l|}
\hline 1 & 1 & 0 & 0 \\
\hline 0 & 2 & 0 & 1 \\
\hline 0 & 0 & 2 & 2 \\
\hline 0 & 1 & 0 & 1 \\
\hline
\end{tabular}

\begin{tabular}{|l|l|l|l|}
\hline 1 & 0 & 0 & 0 \\
\hline 1 & 2 & 0 & 1 \\
\hline 0 & 0 & 2 & 0 \\
\hline 0 & 1 & 2 & 1 \\
\hline
\end{tabular}

\begin{tabular}{|l|l|l|l|}
\hline 0 & 2 & 0 & 0 \\
\hline 0 & 0 & 0 & 1 \\
\hline 1 & 1 & 1 & 1 \\
\hline 0 & 1 & 0 & 0 \\
\hline
\end{tabular}
(b) $v=(1,0)$
(c) $v=(0,1)$
(d) $v=(1,1)$

Fig. 1. Construction of GLCM (a) Original image, (b) GLCM for displacement vector $\mathrm{v}=(1,0)$, (c) GLCM for displacement vector $\mathrm{v}=(0,1),(\mathrm{d})$ GLCM for displacement vector $\mathrm{v}=(1,1)$

The resultant co-occurrence matrix has to be made symmetric by adding the matrix in displacement vector $\mathrm{d}$ with the matrix obtained in $-d$ direction of displacement vector i.e. $W_{d}+W_{-d}$. Some useful texture features that can be derived from GLCM are as follows (Albregtsen 1995).

Energy: it is also known as uniformity or Angular Second Moment (ASM). It provides the sum of squared elements in the Gray level co-occurrence matrix:

$$
\sum_{i} \sum_{j} W_{d}^{2}(i, j) \text {. }
$$

Contrast: this feature measures the local variation in the gray level concurrence matrix:

$$
\sum_{i} \sum_{j}(i-j)^{2} W_{d}(i, j)
$$

Entropy: it is a measure of randomness:

$$
\sum_{i} \sum_{j} W_{d}(i, j) \log W_{d}(i, j) \text {. }
$$

Homogeneity: this feature measures the closeness of the distribution of element in the Gray level co-occurrence matrix to the diagonal of GLCM:

$$
\sum_{i} \sum_{j} \frac{W_{d}(i, j)}{1+|i-j|} .
$$


Dissimilarity: this is similar to contrast but increase linearly. If the local region has the high contrast, the value of dissimilarity is also high.

$$
\sum_{i} \sum_{j} W_{d} \mid i-j
$$

\section{Data resources, instrumentation and study area}

The investigations of present research work have been carried out for the satellite images of different spatial resolution sensors for the Saharanpur district in India and details are as given in the Table 1.

Table 1. Detail of satellite images used for analysis

\begin{tabular}{|c|c|c|c|c|c|}
\hline $\begin{array}{c}\text { S. } \\
\text { No. }\end{array}$ & Satellite & $\begin{array}{c}\text { Image } \\
\text { sensor }\end{array}$ & $\begin{array}{c}\text { Spatial } \\
\text { resolu- } \\
\text { tion }(\mathrm{m})\end{array}$ & $\begin{array}{c}\text { Spectral } \\
\text { resolution }(\mu \mathrm{m})\end{array}$ & $\begin{array}{c}\text { Date of } \\
\text { acqui- } \\
\text { sition }\end{array}$ \\
\hline 1 & $\begin{array}{c}\text { IRS P5 } \\
\text { (Carto- } \\
\text { sat-1) }\end{array}$ & PAN & 2.5 & $0.50-0.85$ & $\begin{array}{c}25 \mathrm{Sept} \\
2010\end{array}$ \\
\hline 2 & $\begin{array}{c}\text { Land- } \\
\text { sat-5 }\end{array}$ & TM & 30 & $\begin{array}{c}0.52-0.61 \\
(\text { Green }) \\
0.63-0.69 \\
(\text { Red) } \\
0.78-0.90 \\
(\mathrm{NIR})\end{array}$ & $\begin{array}{c}10 \text { Sept, } \\
2010\end{array}$ \\
\hline
\end{tabular}

The study area includes Behat and sorrounding areas in Saharanpur district having area central point coordinates $30^{\circ} 10^{\prime} 36^{\prime \prime} \mathrm{N}$ Latitude and $77^{\circ} 35^{\prime} 36^{\prime \prime} \mathrm{E}$ Longitude. Various texture images have been generated in MATLAB software environment. The ERDASImagine version 2013 is also used for the pre-processing and other image analysis tasks.

\section{Methodology}

The methodology for the research work consists of four steps. First step includes the preprocessing of the Panchromatic (PAN) and Multispectral images. The preprocessing of input data is carried out to enhance the appearance and to eliminate the geometric distortions. Secondly, Gray Level Co-occurrence Matrix (GLCM) texture descriptors along with the texture images using different pixel window are obtained from preprocessed images received from previous step. Third step is to produce two images (i) fusion of Multispectral with PAN image (ii) Fusion of PAN with Texture image. Fouth step is to classify the two images along with the PAN image alone by employing the maximum likelihood classification approach for the purpose of topographic object extraction. The adopted methodology for topographic objects extraction is summarized in Figure 2.

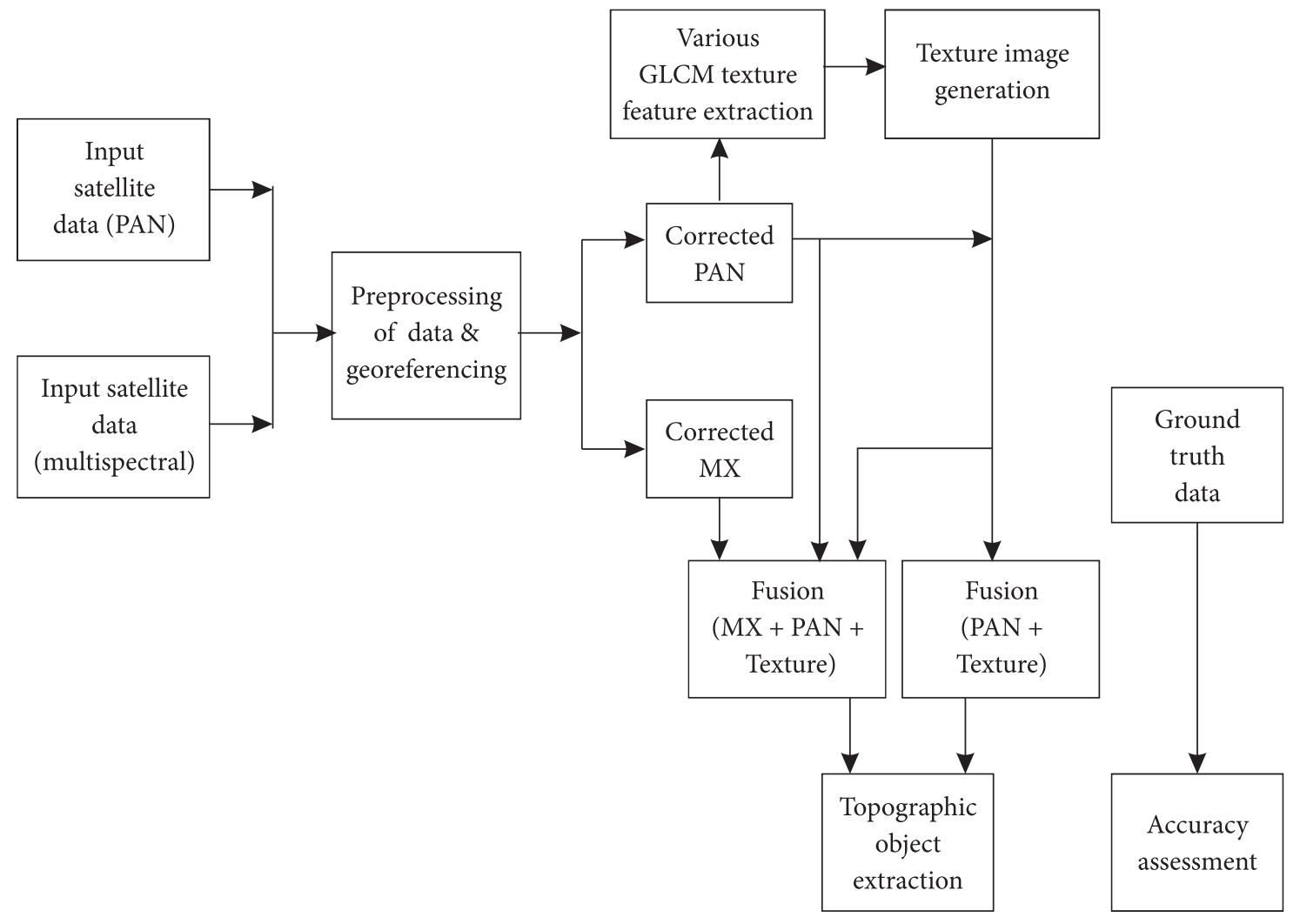

Fig. 2. Methodology 


\section{Results and discussion}

Five texture descriptors are extracted from the Gray Level Co-occurrence Matrix (GLCM) and five texture images of the study area corresponding to each texture descriptor has been generated as shown in Figure 3.

Urban areas are highlighted and appeared as lighter shades of gray where as water bodies in darker shades of gray in contrast and dissimilarity images of texture because both the measures of texture captures the local variation in gray level. On the other hand urban area has darker shades of gray and water bodies in lighter shades of gray in homogeneity and angular second moment texture images.

The effect of using different window size to produce homogeneity texture image has been shown in Figure 4 . The best classification results are obtained when $3 \times 3$ window size is used to produce texture images.

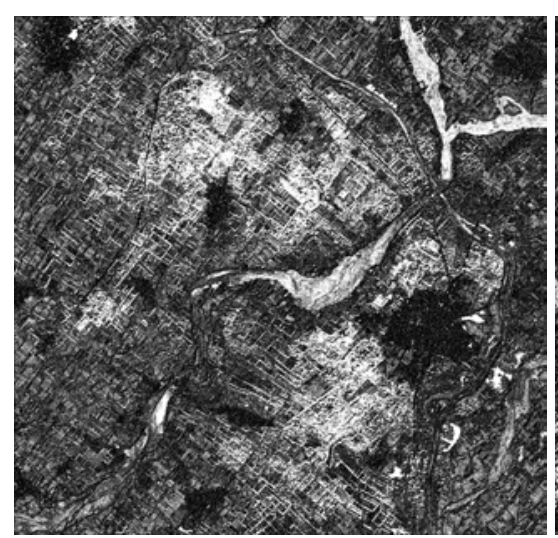

(a) Original PAN image

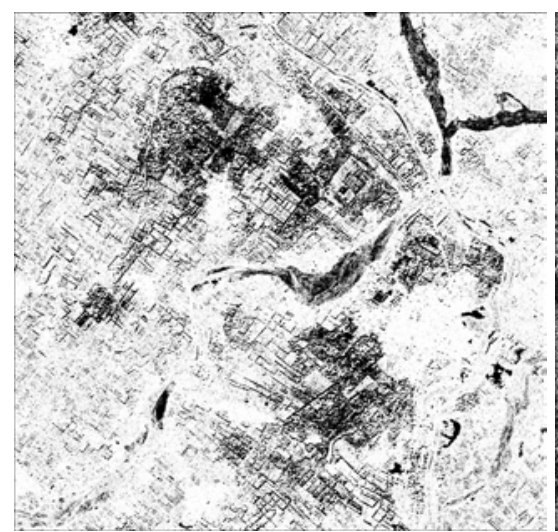

(d) Entropy image

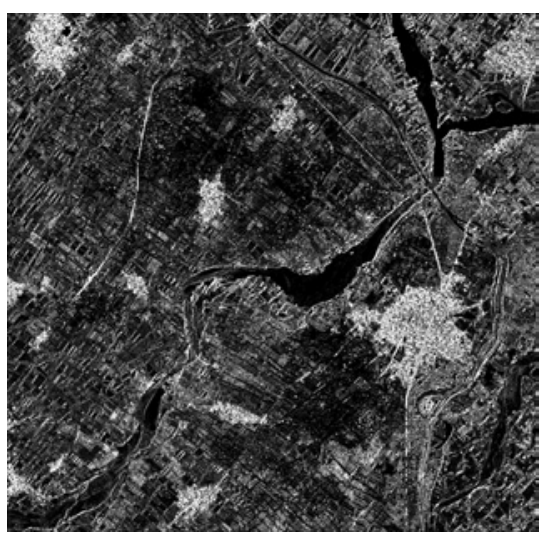

(b) Contrast image

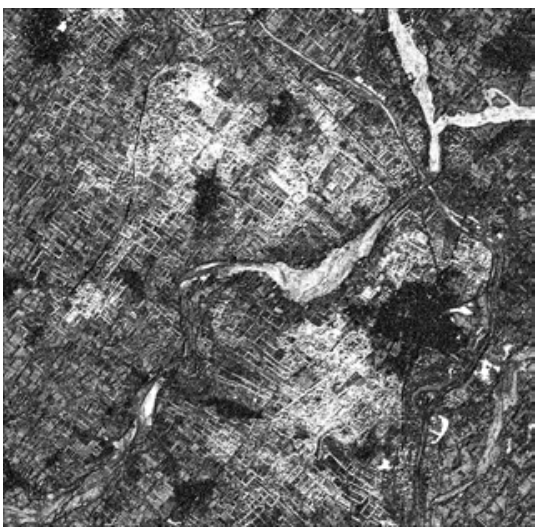

(e) Homogeneity image

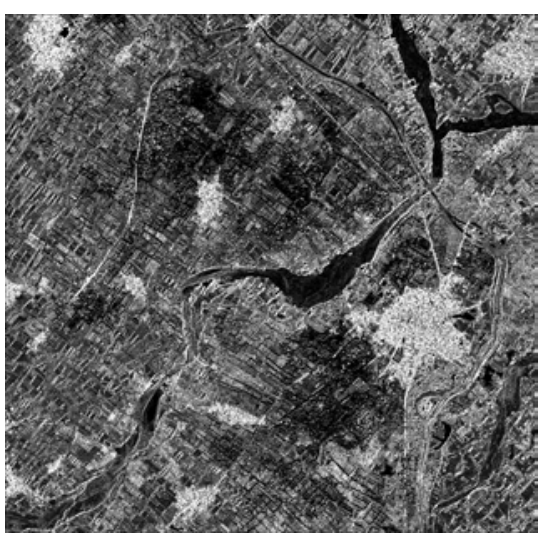

(c) Dissimilarity Image

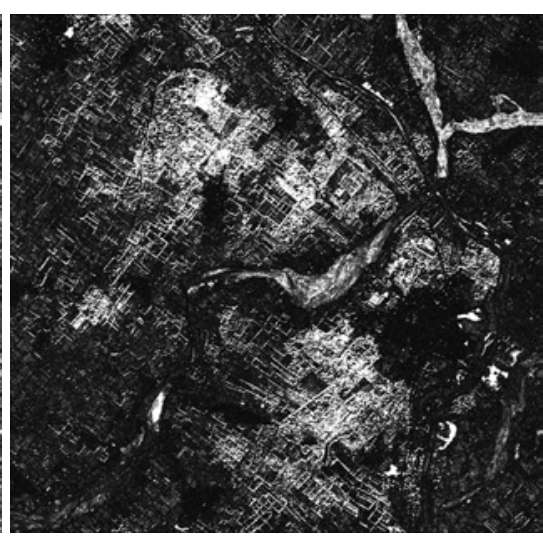

(f) Angular second moment

Fig. 3. Gray level co-occurrence based texture measures

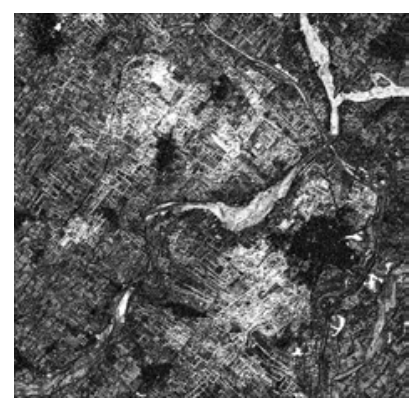

$3 \times 3$

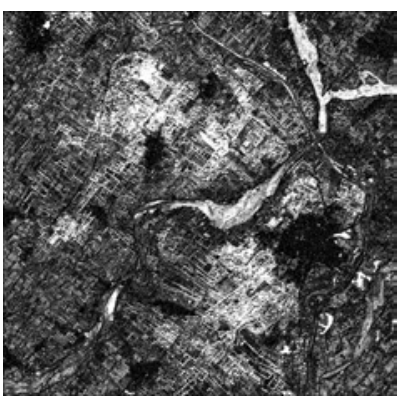

$5 \times 5$

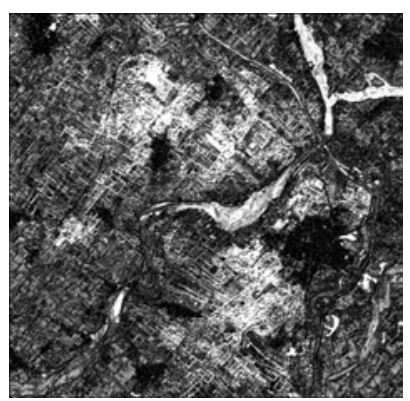

$7 \times 7$

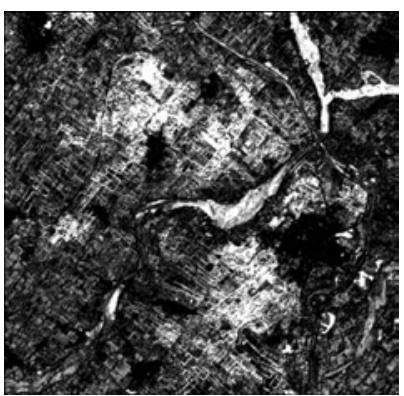

$9 \times 9$

Fig. 4. Homogeneity texture images produced using different window size 


\subsection{Image fusion}

Image fusion is a process combining two or more images to produce a single image with high visual interpretability. On a high spatial resolution panchromatic image (PAN), detailed geometric features can

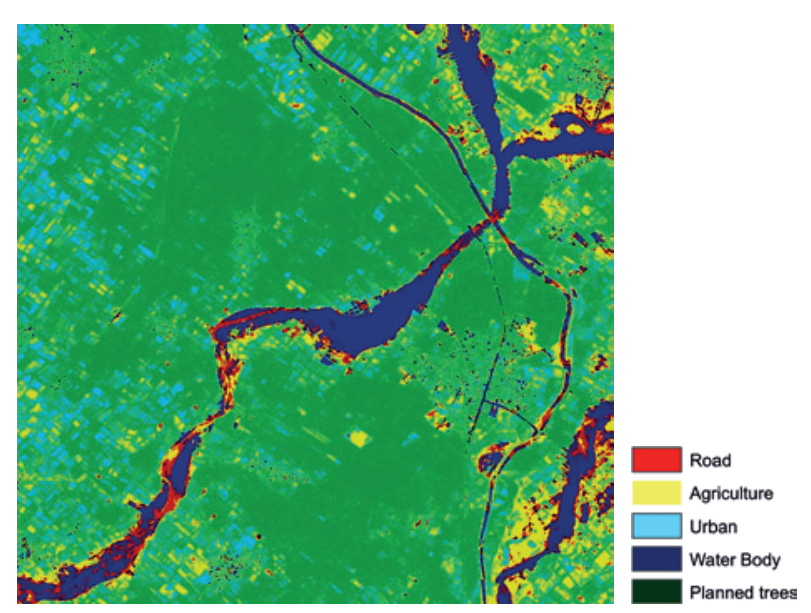

Fig. 5. PAN image classification

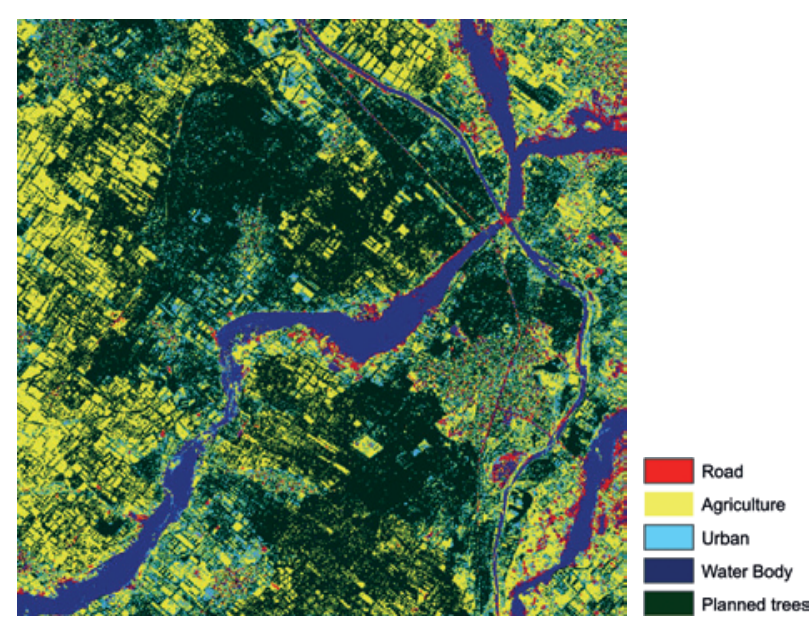

Fig. 6. Fused (PAN + Homogeneity) image classification

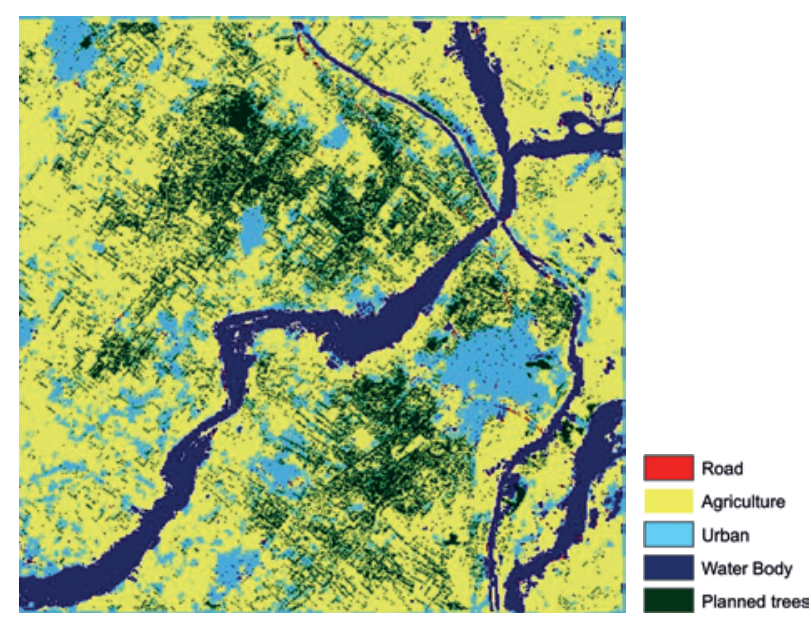

Fig. 7. Fused (PAN + Homogeneity + Multispectral) image classification easily be recognized, while the multispectral images contain affluent spectral information (Shi et al. 2005). The capabilities of the images can be enhanced if the advantages of both high spatial and spectral resolution can be integrated into one single image that can be done using a process known as image fusion. The outcome of image fusion is a new image which is more worthy for human and machine perception or further image-processing tasks such as segmentation, feature extraction and object recognition. Various fusion techniques are available in literature; here we have used resolution merge approach to fuse various images with one another. Resolution merge approach is adopted because it is found best for classification (Yang et al. 2012).

\subsection{Image classification}

Maximum Likelihood Classification (MLC) is considered as one of the most accurate method for image classifications as accepted by various researchers (AlAhmadi, Hames 2009). MLC operates by calculating the probability distribution function for each class present in the image. In our research work, the whole study region has been categorized into five landuse/ cover classes viz. urban, water body, planned trees, roads and agricultural area using MLC method. PAN image has been classified into five classes as shown in Figure 5. Classification results of the PAN image are very poor as most of the classes have got mixed with the other classes (e.g. Road class has mixed with water class and planned trees has mixed with agriculture). Accuracy of the classification has been improved further when classification is carried out on fused image resulting from incorporation of texture band image using same classification method. Water bodies (river and canal), roads and planned trees are well classified with very minute misclassification as depicted from Figure 6. The results of classification are further improved in Figure 7, with the inclusion of multispectral image.

Accuracy assessment of classified images has been conducted with reference to Google Earth. Overall accuracy and Kappa coefficient of classified images are obtained and summarized in Table 2. It has been observed that classification result of the fused images is better than the PAN image alone. In Figure 6, almost all the classes are clearly distinguishable. The increase in overall accuracy is $7.772 \%$ and $12.351 \%$ when texture band and texture plus multispectral bands are fused with the original PAN image respectively. 
Table 2. Accuracy analysis of classified images

\begin{tabular}{|c|l|c|c|}
\hline $\begin{array}{c}\text { S. } \\
\text { No. }\end{array}$ & \multicolumn{1}{|c|}{ Images } & $\begin{array}{c}\text { Overall } \\
\text { accuracy }\end{array}$ & $\begin{array}{c}\text { Kappa } \\
\text { coefficient }\end{array}$ \\
\hline 1 & PAN & $72.6490 \%$ & 0.6144 \\
\hline 2 & PAN + Homogeneity Texture & $80.4213 \%$ & 0.7376 \\
\hline 3 & $\begin{array}{l}\text { Multispectral + PAN + } \\
\text { Texture }\end{array}$ & $85.000 \%$ & 0.8000 \\
\hline
\end{tabular}

\section{Conclusions}

In this paper, the effect of incorporating the texture information along with the image fusion in classification process is discussed. The Gray Level Co-occurrence Matrix based texture measures have been adopted and five texture features are extracted from the Cartosat-1 satellite image. The obtained results indicate that homogeneity texture image generated using $3 \times 3$ window size is best suitable for topographic objects extraction from satellite images. The best classification results with overall accuracy $85.0 \%$ and kappa coefficient 0.80 are obtained when classification is performed on fused image (Multispectral + PAN + Texture).

\section{References}

Al-Ahmadi, F. S.; Hames, A. S. 2009. Comparison of four classification methods to extract land use and land cover from raw satellite images for some remote arid areas, kingdom of Saudi Arabia, Earth 20(1): 167-191.

Albregtsen, F. 1995 Statistical texture measures computed from gray level co-occurrence matrices. Image Processing Laboratory, Department of Informatics, University of Oslo.

Beliakov, G.; James, S.; Troiano, L. 2008. Texture recognition by using GLCM and various aggregation functions, in IEEE International Conference on Computational Intelligence, 1-6 June 2008, Hong Kong. 1472-1476.

Haack, B.; Bechdol, M. 2000. Integrating multisensor data and RADAR texture measures for land cover mapping, Computers \& Geosciences 26(4): 411-421. http://dx.doi.org/10.1016/S0098-3004(99)00121-1

Haala, N.; Brenner, C. 1999. Extraction of buildings and trees in urban environments, Photogrammetric Engineering and Remote Sensing 54: 130-137. http://dx.doi.org/10.1016/S0924-2716(99)00010-6

Kiema, J. B. K. 2002. Texture analysis and data fusion in the extraction of topographic objects from satellite imagery, International Journal of Remote Sensing 23(4): 767-776. http://dx.doi.org/10.1080/01431160010026005
Rogova, G. L.; Stomper, P. C. 2002. Information fusion approach to microcalcification characterization, Information Fusion 3(2): 91-102.

http://dx.doi.org/10.1016/S1566-2535(02)00054-4

Schistad, A. H.; Jain, A. K. 1997. Texture fusion and feature selection applied to SAR imagery, IEEE Transactions on Geoscience and Remote Sensing 35(2): 475-479.

http://dx.doi.org/10.1109/36.563288

Shi, W.; Zhu, C.; Tian, Y.; Nichol, J. 2005. Wavelet-based image fusion and quality assessment, International Journal of Applied Earth Observation and Geoinformation 6: 241-251. http://dx.doi.org/10.1016/j.jag.2004.10.010

Srinivasan, G. N.; Shobha, G. 2008. Statistical texture analysis, in Proceedings of World Academy of Science, Engineering \& Technology, December 2008, 36: 1264-1269. ISSN 20703740.

Wu, Y.; Li, M.; Liao, G. 2007. Multiple features data fusion method in color texture analysis, Applied Mathematics and Computation 185(2): 784-797. http://dx.doi.org/10.1016/j.amc.2006.06.116

Yang, S.; Wang, M.; Jiao, L. 2012. Fusion of multispectral and panchromatic images based on support value transform and adaptive principal component analysis, Information Fusion 13(3): 177-184. http://dx.doi.org/10.1016/j.inffus.2010.09.003

Zhang, Y. 1999. Optimisation of building detection in satellite images by combining multispectral classification and texture filtering, ISPRS Journal of Photogrammetry and Remote Sensing 54: 50-60.

http://dx.doi.org/10.1016/S0924-2716(98)00027-6

Kuldeep, received B.Tech degree in Computer Science and masters degree in Remote Sensing \& GIS from Maulana Azad National Institute of Technology, Bhopal, India. Currently, he is pursuing $\mathrm{PhD}$ in Geomatics from Indian Institute of Technology Roorkee, India. His research interest is Satellite Image Processing, GIS and Remote Sensing.

P. K. GARG, received B.E. in Civil Engg. and M.E. in Advance Surveying \& Photogrammetry from University of Roorkee, India in 1980 and 1982 respectively, and PhD degree in Remote Sensing from University of Bristol, U.K. in 1991. He was a Postdoctoral research fellow in Remote Sensing \& GIS at University of Reading, U.K. He is currently a Professor in Geomatics Division, Civil Engineering Department, Indian Institute of Technology Roorkee, India. His research interest is Satellite Image Analysis, Landuse Mapping, Digital Image Processing, GPS Survey and GIS. 